\title{
A detailed spectroscopy of the carbon-rich star BD $+57^{\circ} 2161$
}

\author{
L. Začs ${ }^{1}$, M. R. Schmidt ${ }^{2}$, F. A. Musaev ${ }^{3,4,5}$, G. A. Galazutdinov ${ }^{6,3}$, and J. Sperauskas ${ }^{7}$ \\ ${ }^{1}$ Institute of Atomic Physics and Spectroscopy, University of Latvia, Raiņa bulvāris 19, 1586 Rīga, Latvia \\ e-mail: zacs@latnet.lv \\ 2 N. Copernicus Astronomical Center, ul. Rabiańska 8, 87-100 Toruń, Poland \\ e-mail: schmidt@ncac. torun.pl \\ 3 Special Astrophysical Observatory and Isaac Newton Institute of Chile, SAO Branch, Nizhnij Arkhyz, 369167, Russia \\ e-mail: faig@sao.ru, gala@sao.ru \\ ${ }^{4}$ ICAMER, National Academy of Sciences of Ukraine, 361605 Peak Terskol, Kabardino-Balkaria, Russia \\ 5 Shamakhy Astrophysical Observatory, National Academy of Sciences of Azerbaijan, Azerbaijan \\ ${ }^{6}$ Korean Astronomy Observatory, Optical Astronomy Division, 61-1, Whaam-Dong, Yuseong-Gu, Daejon, 305-348, Korea \\ e-mail: gala@boao.re.kr \\ 7 Vilnius University Observatory, Ciurlionio 29, Vilnius 2009, Lithuania \\ e-mail: julius.sperauskas@ff.vu.lt
}

Received 23 November 2004 / Accepted 9 May 2005

\begin{abstract}
An LTE abundance analysis based on high-resolution spectra is presented for the carbon-rich star BD $+57^{\circ} 2161$, whose evolutionary status is unknown. With $[\mathrm{C} / \mathrm{Fe}]=+0.4 \mathrm{dex}$ and a mean $s$-process overabundance of $[\mathrm{s} / \mathrm{Fe}] \simeq+1.5 \mathrm{dex}$ the peculiar atmospheric composition of $\mathrm{BD}+57^{\circ} 2161$ is confirmed. The ${ }^{12} \mathrm{C} /{ }^{13} \mathrm{C}$ abundance ratio was found to be about 10 . The mild iron deficiency, $[\mathrm{Fe} / \mathrm{H}]=-0.2$, supports the idea that $\mathrm{BD}+57^{\circ} 2161$ could be an old-disk-population object. Radialvelocity measurements confirm the binary nature of the star. Therefore the peculiar chemical composition could be due to the mass transfer from the secondary - AGB star in the past. Orbital parameters are estimated for another star of this group, $\mathrm{BD}+75^{\circ} 348$
\end{abstract}

Key words. stars: evolution - stars: atmospheres - stars: abundances - stars: chemically peculiar stars: individual: $\mathrm{BD}+57^{\circ} 2161$ - stars: individual: $\mathrm{BD}+75^{\circ} 348$

\section{Introduction}

Carbon stars are recognizable in the optical spectral region by the presence of absorption bands of carbon bearing molecules $\left(\mathrm{C}_{2}, \mathrm{CN}, \mathrm{CH}, \mathrm{C}_{3}, \mathrm{SiC}\right.$, etc. $)$ and are chemically characterized by the presence of $\mathrm{C} / \mathrm{O}>1$. The excess of carbon in such stars is the result of stellar nucleosynthesis in the star itself or in a binary companion. The $s$-process elements are enhanced usually by factors of 10-100. Stars with enhanced carbon-bearing features come in many spectral types: the N stars, the R stars, barium stars, CH stars, dwarf carbon stars, and others (for a review, see Wallerstein \& Knapp 1998). However, very often one given star is classified by different authors in different spectral types. The task of high-resolution spectroscopy is to identify the chemical peculiarities with particular evolutionary paths.

The "CH-like stars" were first recognized as a distinct class among the ordinary carbon stars of early spectral types by Yamashita (1972) using low-resolution spectra. Sixteen stars were selected from the group of $\mathrm{C} 0-\mathrm{C} 3$ stars which display a definite enhancement of Ba II line at $4554 \AA$, a low radial velocity, and proper motion (Yamashita 1975). These stars' spectra closely resemble those of classical $\mathrm{CH}$ stars. However, the $\mathrm{CH}$ stars have large radial velocities (RV), typically $\sim 200 \mathrm{~km} \mathrm{~s}^{-1}$, indicating that they are halo objects, while the ordinary $\mathrm{C} 0-\mathrm{C} 3$ and $\mathrm{CH}$-like stars are concentrated in the range of $R V<60 \mathrm{~km} \mathrm{~s}^{-1}$. It is probable from these velocity distributions that the $\mathrm{CH}$ stars and the $\mathrm{CH}$-like stars are from different stellar populations, and that the $\mathrm{CH}$-like stars form a single natural group. However, their real nature is rather uncertain at this time (see Wallerstein \& Knapp 1998). An abundance analysis for one star of this group, BD $+75^{\circ} 348$, revealed a large enhancement of carbon and $s$-process elements (Začs et al. 2000). The modest iron deficiency, $[\mathrm{Fe} / \mathrm{H}]=-0.8$, and the kinematic data support the idea that $\mathrm{BD}+75^{\circ} 348$ is an old disk population object. Therefore, $\mathrm{CH}$-like stars could be analogues of barium stars in the old disk population. The program of high-resolution spectroscopy and radial velocity monitoring was launched to clarify the evolutionary status of these stars. Here the results of detailed spectroscopy are presented for the second star of this group, BD $+57^{\circ} 2161$.

$\mathrm{BD}+57^{\circ}$ 2161 (=CGCS $4784=$ HIP 99725) has been classified before as R0 (Vandervort 1958), C4,1 ch (Yamashita 1975), C3,1 (Yamashita et al. 1977), and C1,1 CH (Bartkevicius \& Lazauskaite 1996). Yamashita $(1972,1975)$ noted a low $R V_{\odot}$ of $-47 \mathrm{~km} \mathrm{~s}^{-1}$ and low proper motion for this object in spite of the enhanced Ba II line at $4554 \AA$. The star is suspected to be a 

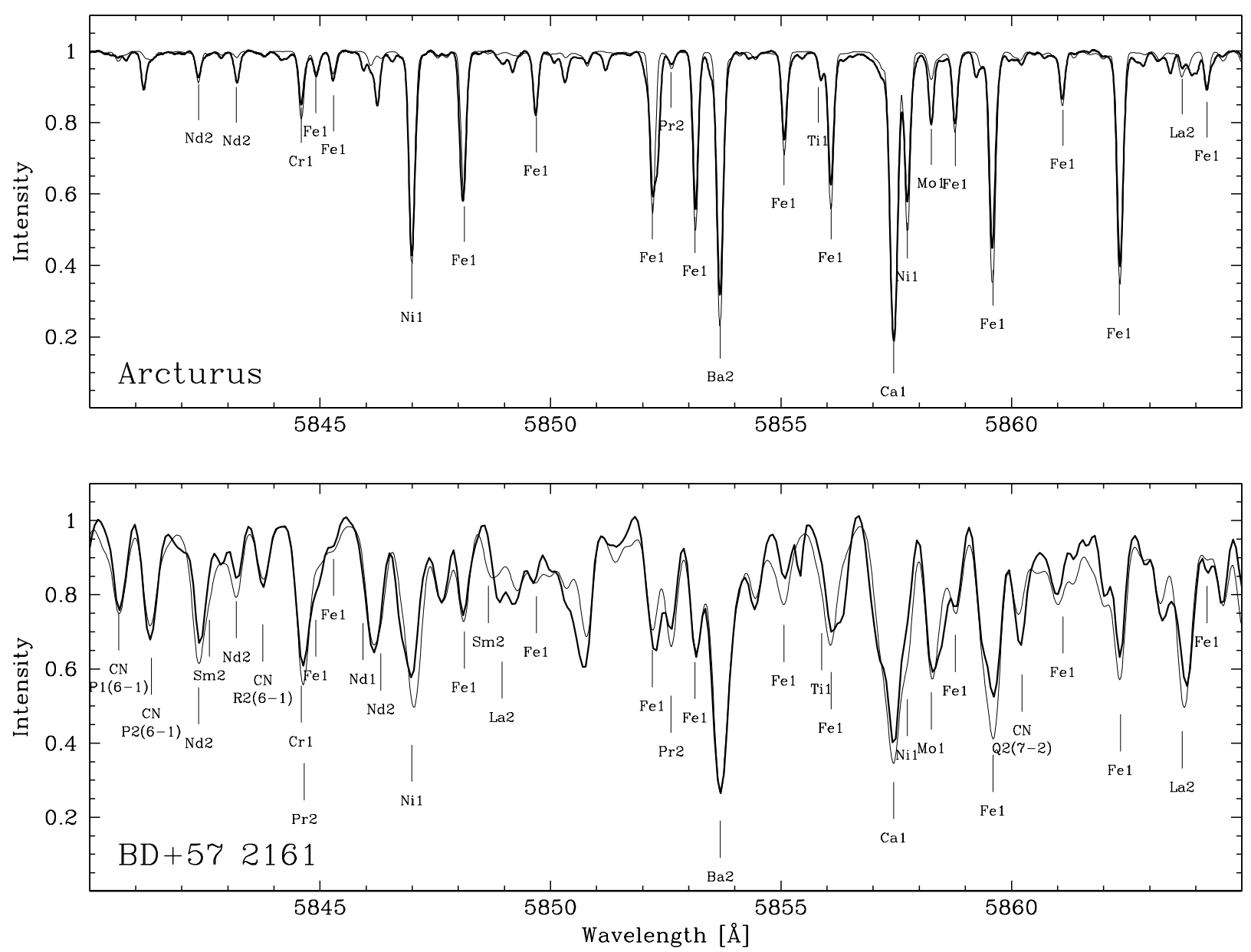

Fig. 1. Observed (thick line) and synthetic spectra of $\mathrm{BD}+57^{\circ} 2161$ around the Ba II line at $5853.68 \AA$. The synthetic photospheric spectrum was generated using the atmospheric model with $T_{\text {eff }}=4555 \mathrm{~K}, \log g=2.5(\mathrm{cgs}), \xi_{t}=2.0 \mathrm{~km} \mathrm{~s}^{-1}$, and enhanced abundances for the neutron-capture elements (Table 3). Also shown is the spectrum for the standard star Arcturus (Hinkle et al. 2000).

variable (NSV 12948), although significant variability is absent; and the visual magnitude ranges from 9.6 to 9.75 (Vandervort 1958; Yamashita 1975; ESA 1997). Bolometric magnitude $M_{\text {bol }}=-1.53$ estimated by Bergeat et al. (2002) is typical of R-type carbon stars (Knapp et al. 2001). Investigations of the chemical and isotopic peculiarities in such a star encourage improvements in stellar evolutionary models and the theory of nucleosynthesis and convection.

\section{Observations and reduction}

A high-resolution spectrum for $\mathrm{BD}+57^{\circ} 2161$ was obtained with the coudé échelle spectrometer MAESTRO fed by the $2 \mathrm{~m}$ telescope at the Observatory on the Terskol Peak in Northern Caucasus equipped with a CCD detector (Musaev et al. 1999) with a resolving power of $\sim 45000$. A total exposure of $7200 \mathrm{~s}$ was made on 19 November 2002 . The spectrum covered a spectral region from 3600 to $10200 \AA$ in 85 wavelength bands overlapping shortward of $\mathrm{H}_{\alpha}$. Each region spanned from 50 to $140 \AA$. Unfortunately owing to the energy distribution in cool stars, the $\mathrm{S} / \mathrm{N}$ ratio in the blue region is low. Therefore only the red and near-infrared spectral regions were used for abundance calculations. The resulting rectified spectrum for one region, along with the synthesized one, is presented in Fig. 1; also shown are the observed (Hinkle et al. 2000) and calculated spectra for the standard star Arcturus.

Radial-velocity monitoring of $\mathrm{BD}+57^{\circ} 2161$ was started in 2003 by one of us (J.S.) using the CORAVEL spectrometer (Upgren et al. 2002) of the Vilnius University installed on the $1.65 \mathrm{~m}$ telescope at the Moletai Observatory (Lithuania) and a $1.5 \mathrm{~m}$ telescope at the Steward Observatory (Arizona, Mount Lemmon). Eight $R V$ measurements were made in a 114 day time spread (Table 1) with the standard deviation of one measurement of $0.7 \mathrm{~km} \mathrm{~s}^{-1}$. Another star, BD $+75^{\circ} 348$, from the group of "CH-like stars" was monitored by Roger Griffin with a radial-velocity spectrometer at the coudé focus of the Cambridge 36-inch reflector. Thirty-three RV measurements were carried out (Table 2) with the standard deviation of one measurement of $0.4 \mathrm{~km} \mathrm{~s}^{-1}$. To bring the zero-points into agreement, $0.8 \mathrm{~km} \mathrm{~s}^{-1}$ was subtracted from the Cambridge measurements.

\section{Analysis}

A qualitative inspection of the high-resolution spectrum for $\mathrm{BD}+57^{\circ} 2161$ shows that, compared to the moderately metal poor $([\mathrm{Fe}]=-0.5)$ giant Arcturus $(\mathrm{K} 1.5 \mathrm{III})$, the lines of the iron-peak elements are of similar strength (see, for example, 
Table 1. Radial-velocity observations of BD $+57^{\circ} 2161$.

\begin{tabular}{rrc}
\hline \hline Date & MJD & $\begin{array}{c}\text { Velocity } \\
\left(\mathrm{km} \mathrm{s}^{-1}\right)\end{array}$ \\
\hline 2003 June 19.01 & 2452839.01 & -65.9 \\
19.99 & 839.99 & -65.8 \\
Aug. 12.88 & 863.88 & -68.2 \\
13.90 & 864.90 & -66.7 \\
18.91 & 869.91 & -67.5 \\
Oct. 17.11 & 930.11 & -68.2 \\
Nov. 5.16 & 949.16 & -67.5 \\
9.11 & 953.10 & -68.1 \\
\hline
\end{tabular}

Fig. 1), while features due to $\mathrm{C}_{2}, \mathrm{CN}$, and neutron-capture elements are enhanced. For example, the barium line at $5853.68 \AA$ in the spectrum of $\mathrm{BD}+57^{\circ} 2161$ is very strong $(E W=344 \mathrm{~m} \AA)$ in comparison with that for Arcturus (123 m $\AA$ ), indicating a significant barium overabundance. Notice that the typical R0-R3 stars show weak molecular bands, and their atomic-line spectra are equivalent to G9-K2 normal giants; however, the s-process elements usually are not enhanced (Dominy 1984). Notice, too, that the widths $(F W H M)$ of absorption lines are larger than the instrumental profile $(0.15 \AA)$ owing to macroturbulence and/or rotation of the star. Our calculations, however, indicate that in the case of rotation the velocity should be lower than $5 \mathrm{~km} \mathrm{~s}^{-1}$. The most prominent molecular feature for $\mathrm{BD}+57^{\circ} 2161$ in the observed region is the $\mathrm{C}_{2}$ Swan system band $(0,0)$ at $5165 \AA$ with a total equivalent width of the head of $\simeq 2.5 \AA$. Clearly visible in $\mathrm{BD}+57^{\circ} 2161$ are also the lines of another $C_{2}$ Swan system. Besides, over all the analyzed spectrum, bands of the $\mathrm{CN}$ red system are evident, blending the atomic absorption lines significantly. Although continuum definition becomes an increasingly serious problem for the later spectral types, the spectral synthesis of the atomic and molecular spectrum for $\mathrm{BD}+57^{\circ} 2161$ over the entire observed spectral region gives evidence that the continuum redward of $4800 \AA$ is in general clearly defined. However, some of the atomic lines are significantly blended with molecular ones, especially owing to the open structure of the $\mathrm{CN}$ red system lines. The final selection of clean atomic absorption lines for abundance analysis was done using the synthesized atomic and molecular spectrum.

\subsection{Radial velocities}

\subsection{1. $\mathrm{BD}+57^{\circ} 2161$}

The radial velocity for $\mathrm{BD}+57^{\circ} 2161$ was measured from the high-resolution MAESTRO spectrum (JD2 452 598) and a number of symmetric atomic absorption lines selected over all the spectral region. The mean $R V_{\odot}$ was found to be $-56.2 \pm$ $0.5 \mathrm{~km} \mathrm{~s}^{-1}$. A correction of zero-point was made by the use of terrestrial absorption lines in the observed spectrum, $\Delta R V=$ $+0.15 \mathrm{~km} \mathrm{~s}^{-1}$. According to the measurements obtained with the CORAVEL of the Vilnius University (Table 1), the mean radial velocity was found to be $-67.2 \pm 0.4 \mathrm{~km} \mathrm{~s}^{-1}$. Therefore the binarity of $\mathrm{BD}+57^{\circ} 2161$ is suspected. However, more
Table 2. Radial-velocity observations of BD $+75^{\circ} 348$.

\begin{tabular}{rrrrc}
\hline \hline Date & MJD & $\begin{array}{r}\text { Velocity } \\
\left(\mathrm{km} \mathrm{s}^{-1}\right)\end{array}$ & Phase & $\begin{array}{c}(\mathrm{O}-\mathrm{C}) \\
\left(\mathrm{km} \mathrm{s}^{-1}\right)\end{array}$ \\
\hline 1994 May 27.90 & 49499.00 & 43.3 & $\overline{3} .957$ & 0.0 \\
2000 Nov. 13.23 & 51861.23 & 56.8 & 0.225 & -0.6 \\
30.19 & 878.19 & 57.1 & .241 & -0.4 \\
Dec. 22.10 & 900.10 & 57.2 & .262 & -0.2 \\
2001 Jan. 11.12 & 51920.12 & 57.5 & 0.281 & +0.1 \\
Apr. 28.88 & 52027.88 & 57.1 & 0.385 & +0.7 \\
Nov. 1.16 & 214.16 & 52.6 & .563 & -0.6 \\
14.23 & 227.23 & 52.5 & .576 & -0.4 \\
Dec. 14.17 & 257.17 & 51.4 & .605 & -0.8 \\
2002 Jan. 1.11 & 52275.11 & 51.8 & 0.622 & 0.0 \\
Feb. 4.11 & 309.11 & 51.3 & .655 & +0.4 \\
Mar. 1.03 & 334.03 & 51.0 & .679 & +0.7 \\
Apr. 3.93 & 367.93 & 49.0 & .711 & -0.3 \\
May 4.89 & 398.89 & 48.5 & .741 & +0.1 \\
Sep. 28.19 & 545.19 & 43.5 & .881 & -0.1 \\
Oct. 18.21 & 565.21 & 43.3 & .901 & +0.2 \\
Dec. 5.13 & 613.13 & 43.0 & .947 & -0.1 \\
2003 Jan. 5.13 & 52644.13 & 44.5 & .976 & +0.3 \\
Feb. 15.06 & 685.06 & 46.8 & 1.016 & -0.4 \\
Mar. 3.00 & 701.00 & 48.4 & .031 & -0.2 \\
23.92 & 721.92 & 50.6 & .051 & +0.1 \\
Apr. 7.92 & 736.92 & 51.6 & .065 & -0.1 \\
May 6.93 & 765.93 & 54.6 & .093 & +0.9 \\
Sep. 18.18 & 900.18 & 56.9 & .222 & -0.5 \\
Oct. 18.22 & 930.22 & 57.5 & .251 & 0.0 \\
Nov. 28.14 & 971.14 & 57.1 & .290 & -0.2 \\
Dec. 27.18 & 53000.18 & 57.2 & .318 & +0.1 \\
2004 Jan. 17.13 53021.13 & 57.4 & 1.338 & +0.5 \\
Feb. 23.03 & 058.03 & 56.6 & .374 & +0.1 \\
Mar. 16.98 & 080.98 & 56.4 & .396 & +0.2 \\
Apr. 13.93 & 108.93 & 56.6 & .422 & +0.8 \\
May 10.88 & 135.88 & 55.5 & .448 & +0.1 \\
Sep. 21.18 & 269.18 & 52.5 & .576 & -0.4 \\
Oct. 19.22 & 297.22 & 52.4 & .603 & +0.1 \\
\hline & & & & \\
\hline
\end{tabular}

observations with a larger time spread are needed to calculate orbital parameters.

\subsection{2. $\mathrm{BD}+75^{\circ} 348$}

For BD $+75^{\circ} 348$, using 34 measurements available (Table 2), Griffin calculated the folowing orbital elements: $P=1042 \pm$ 5 days, $T=$ MJD $52669 \pm 7, \gamma=51.66 \pm 0.08 \mathrm{~km} \mathrm{~s}^{-1}$, asini $=97.9 \pm 2.0 \mathrm{Gm}, K=7.29 \pm 0.13 \mathrm{~km} \mathrm{~s}^{-1}, f(m)=$ $0.0346 \pm 0.0021 M_{\odot}, e=0.347 \pm 0.018, \omega=233.7 \pm 2.9 \mathrm{deg}$, rms residual $=0.40 \mathrm{~km} \mathrm{~s}^{-1}$. The observed radial velocities of $\mathrm{BD}+75^{\circ} 348$ plotted as a function of phase are shown in Fig. 2. The calculated orbital elements are typical of extrinsic carbonrich stars (see McClure \& Woodsworth 1990).

\subsection{Atmospheric parameters}

The effective temperature of $\mathrm{BD}+57^{\circ} 2161$ was estimated by Bergeat et al. (2001) using intrinsic spectral energy distribution and the atmospheric models technique, $T_{\text {eff }}=4555 \mathrm{~K}$. Using 
Table 3. Averaged absolute and relative abundances for $\mathrm{BD}+57^{\circ}$ 2161. The standard deviations and the numbers of lines used in the analysis are also given. The last column indicates the systematic errors produced by the uncertainty in $\Delta T_{\text {eff }}=-200 \mathrm{~K}$.

\begin{tabular}{|c|c|c|c|c|c|}
\hline Element $(\mathrm{X})$ & $\log \epsilon(\mathrm{X})$ & $\sigma$ & $n$ & {$[\mathrm{X} / \mathrm{Fe}]$} & $\Delta[X / F e]$ \\
\hline C I & 8.80 & & 1 , syn & +0.5 & +0.21 \\
\hline $\mathrm{N}_{\mathrm{I}}$ & 8.22 & & syn & +0.5 & \\
\hline O I & 8.45 & & 1 & -0.2 & +0.28 \\
\hline $\mathrm{Na}$ I & 6.06 & 0.07 & 4 & -0.1 & +0.21 \\
\hline Mg I & 7.34 & 0.12 & 2 & 0.0 & +0.14 \\
\hline $\mathrm{Si}$ I & 7.65 & & 1 & +0.3 & +0.14 \\
\hline$S_{I}$ & 7.26 & & 1 & +0.1 & +0.18 \\
\hline K I & 4.87 & & 1 & 0.0 & +0.04 \\
\hline $\mathrm{Ca} \mathrm{I}$ & 6.08 & 0.08 & 5 & -0.1 & +0.10 \\
\hline Ca II & 6.20 & & 1 & 0.0 & +0.18 \\
\hline Sc I & 2.85 & & 1 & -0.1 & +0.03 \\
\hline Sc II & 3.29 & 0.10 & 3 & +0.3 & -0.12 \\
\hline Ti I & 5.17 & 0.07 & 14 & +0.4 & -0.20 \\
\hline Ti II & 5.28 & 0.07 & 4 & +0.4 & -0.08 \\
\hline $\mathrm{V}_{\mathrm{I}}$ & 3.96 & 0.10 & 6 & +0.2 & +0.19 \\
\hline $\mathrm{Cr} \mathrm{I}$ & 5.23 & 0.10 & 7 & -0.2 & -0.02 \\
\hline $\mathrm{Fe} \mathrm{I}$ & 7.29 & 0.18 & 52 & 0.0 & - \\
\hline $\mathrm{Fe}$ II & 7.32 & 0.05 & 4 & 0.0 & - \\
\hline $\mathrm{Ni} I$ & 6.14 & 0.12 & 4 & +0.1 & -0.04 \\
\hline $\mathrm{Rb}$ I & 2.85 & & 1 & +0.5 & -0.12 \\
\hline Sr I & 4.01 & 0.05 & 2 & +1.3 & -0.06 \\
\hline Y I & 3.54 & 0.07 & 3 & +1.5 & -0.23 \\
\hline Y II & 3.70 & 0.14 & 6 & +1.6 & -0.08 \\
\hline $\mathrm{ZrI}$ & 3.39 & 0.07 & 7 & +1.0 & -0.22 \\
\hline $\mathrm{Zr}$ II & 3.38 & & 1 & +1.0 & -0.18 \\
\hline Mo I & 3.30 & 0.04 & 2 & +1.6 & -0.17 \\
\hline Ru I & 2.61 & & 1 & +1.0 & -0.26 \\
\hline Ba II & 3.50 & 0.22 & 3 & +1.6 & -0.12 \\
\hline La II & 2.84 & 0.05 & 3 & +1.9 & -0.17 \\
\hline Ce II & 2.91 & 0.14 & 4 & +1.5 & -0.05 \\
\hline Pr II & 2.47 & 0.01 & 2 & +1.9 & -0.12 \\
\hline Nd II & 3.13 & 0.09 & 11 & +1.8 & -0.17 \\
\hline
\end{tabular}

infrared JHK colors and two different calibrations, Ulla et al. (1997) obtained lower temperatures, 4087 and $>4200$ K. For abundance calculations in this paper we accepted the higher temperature. An excitation analysis of the Fe I lines in general supports this value (see Začs et al. 1998). The surface gravity $(\log g)$ was determined from the Fe I/Fe II ionization balance and the microturbulent velocity by forcing the abundances of individual Fe I lines to be independent of equivalent width. The resulting atmospheric parameters for $\mathrm{BD}+57^{\circ} 2161$ are as follows: $T_{\text {eff }}=4555 \mathrm{~K}, \log g=2.5(\mathrm{cgs})$, and $\xi_{t}=2.0 \mathrm{~km} \mathrm{~s}^{-1}$, approximately equal to those for normal $\mathrm{K}$ giants (Gustafsson \& Bell 1979).

\subsection{The abundance analysis}

The standard LTE line analysis program WIDTH9 developed by Kurucz was employed, and the model atmospheres were extracted from Kurucz (1993). Spectral lines stronger than $250 \mathrm{~m} \AA$ were not used for abundance calculations. The clean lines (Table 3 ) were selected by successive iteration using the

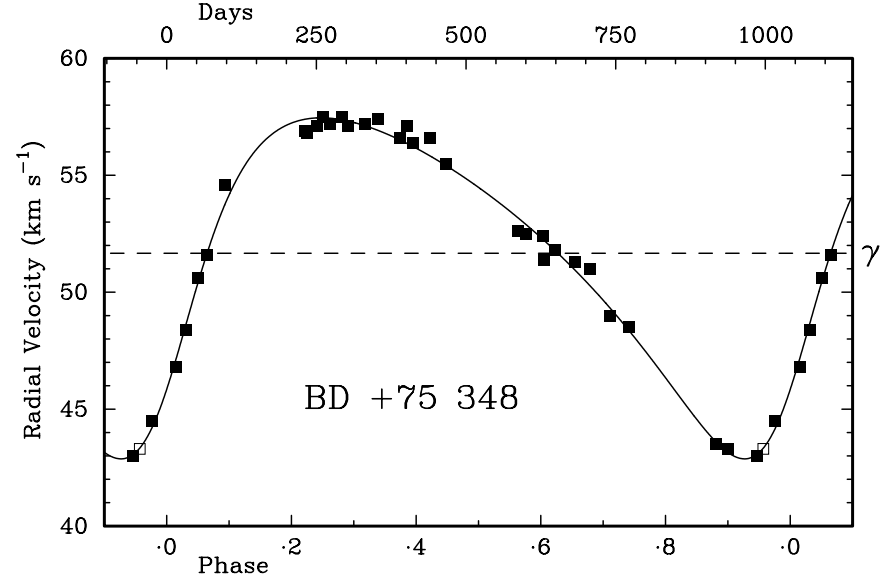

Fig. 2. The observed radial velocities of $\mathrm{BD}+75^{\circ} 348$ plotted as a function of phase, with the velocity curve corresponding to the adopted orbital elements drawn through them. All measurements except one (open square; Začs et al. 2000) were made with the Cambridge Coravel.

calculated atomic and molecular spectrum over the entire spectral region. The synthetic spectra were generated by the spectral synthesis code developed by one of us (M.S.). The $\mathrm{C}_{2}$ Swan system $(0,1)$ bandhead at $5635 \AA$ and band at $5585 \AA$ were used to determine the carbon abundance for $\mathrm{BD}+57^{\circ} 2161$ (Figs. 3 and 4 ). Since the $\mathrm{C}_{2}$ band is not fixed by the carbon abundance alone (the $\mathrm{CO}$ molecule determines the partial pressures of free carbon and oxygen so the $\mathrm{C}_{2}$ band depends on both the $\mathrm{C}$ and $\mathrm{O}$ abundances), an independent estimate of the oxygen abundance is needed. Unfortunately, only one useful oxygen line (7771.96 $\AA$; $10.1 \mathrm{m \AA} ; \log g f=0.29)$ was found for abundance analysis; other O I lines are blended by $\mathrm{CN}$ lines. Therefore, the oxygen abundance was fixed at the value of $[\mathrm{O} / \mathrm{Fe}]=-0.17$. The nitrogen abundance was found to be $\log \epsilon(\mathrm{N})=8.22$ on the basis of spectrum synthesis of $\mathrm{CN}(6,1)$ lines (see Fig. 1). In order to obtain reliable estimates of the carbon isotope ratio, an analysis of selected $\mathrm{CN}$ red system $(2,0)$ lines near $7980 \AA$ was made. The spectrum synthesis of this region indicates that ${ }^{12} \mathrm{C} /{ }^{13} \mathrm{C}$ abundance ratio for $\mathrm{BD}+57^{\circ} 2161$ is about 10 .

The code for the spectrum synthesis adopts atomic and molecular partition functions and the continuous opacity package from ATLAS9 (Kurucz 1992). The ionization and dissociation equilibrium was calculated with the usage of Tsuji's (1973) dissociative equilibrium molecular constants. A dissociation energy of $6.25 \mathrm{eV}$ was adopted for $\mathrm{C}_{2}$ (Cooper 1981) following Vanture (1992c). As a primary source of atomic data the VALD database (Piskunov et al. 1995) was used. The final list of lines was carefully selected by comparison with the NIST database (Kelleher et al. 1999). The list of molecular lines of $C_{2}$ and CN was adopted from Kurucz's database (Kurucz 1993). In addition SCAN-CN tape (Jørgensen \& Larsson 1990) was used for comparison purposes. Notice the systematic difference in $\log g f$ and wavelengths for ${ }^{13} \mathrm{CN}(2,0)$ lines. Jørgensen \& Larsson (1990) recommend the multiplication of their gf values with a factor of 0.734 to agree with those given by Knowles et al. (1988) and Bauschlicher et al. (1988). 

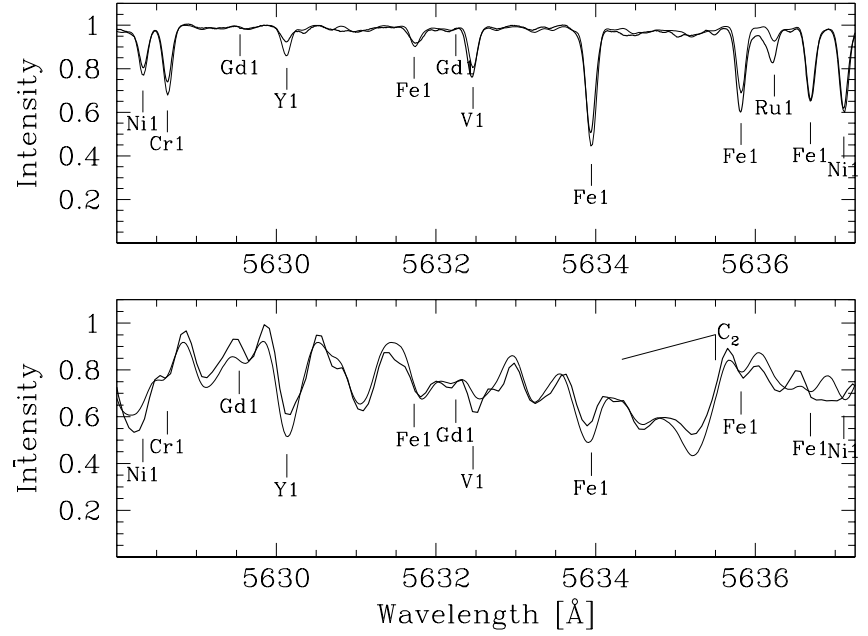

Fig. 3. The observed and synthetic spectra of the CH-like star $\mathrm{BD}+57^{\circ} 2161$ (bottoms) around the $\mathrm{C}_{2}$ Swan system $(0,1)$ bandhead at $5635 \AA$. The thin line represents the synthetic spectrum with carbon abundances of $\log \epsilon(\mathrm{C})=8.80$. Also shown is the spectrum for the standard star Arcturus (top).

The systematic errors produced in abundances by uncertainties in $T_{\text {eff }}( \pm 200 \mathrm{~K}), \log g( \pm 0.3 \mathrm{dex})$, and $\xi_{t}\left( \pm 0.5 \mathrm{~km} \mathrm{~s}^{-1}\right)$ would lead to errors less than $0.3 \mathrm{dex}$, a little worse than those for barium stars with similar effective temperatures (see Začs et al. 1997). However, the variation in temperature by $-200 \mathrm{~K}$ leads to the variation in $\log g$ of about -0.7 dex. The final error estimate (Table 3; last column) includes the $T_{\text {eff }}$ and $\log g$ coupling term. $\Delta[\mathrm{Fe} / \mathrm{H}]$ was found to be -0.15 dex. To check this methodology our result for the CH star HD 201626 was compared with that derived by Vanture (1992a), and reasonable agreement $(\log \epsilon(\mathrm{C})=8.55$ and 8.4, respectively) was found.

Large enhancements of $s$-process elements in the atmosphere of $\mathrm{BD}+57^{\circ} 2161$ are found relative to the solar values: $[\mathrm{s} / \mathrm{Fe}] \simeq+1.5$ dex on the average. The fit between the observed and synthesized spectra confirms a mild iron deficiency along with the overabundance of neutron-capture elements (see, for example, Fig. 1). The heavy $s$-process elements are relatively more enhanced than the light $s$-process peak elements, $[\mathrm{h} s / 1 s] \simeq+0.4 \mathrm{dex}$. Spectral synthesis of the molecular band provides enhanced carbon abundance for $\mathrm{BD}+57^{\circ} 2161$, $[\mathrm{C} / \mathrm{Fe}]=+0.4 \mathrm{dex}$, which suggests that material rich in carbon and neutron-capture elements has been added to the envelope of this star. Although the $S / N$ ratio shortward of $4800 \AA$ is low and the spectrum is very crowded, we also attempted to check in our spectrum the presence of $\mathrm{Pb}$ I lines at 4019, 4057, and $4062 \AA$. Blending in this region makes the detection of weak features difficult; however, a medium strong line is seen at $4057.81 \AA$ in the spectrum of $\mathrm{BD}+57^{\circ} 2161$ (Fig. 5). Comparison with the identification provided by Van Eck et al. (2001) for the metalpoor star HD 196944 indicates clearly that this feature is not due to the $\mathrm{CH}$ molecule. Thus a significant enhancement of lead in the atmosphere of $\mathrm{BD}+57^{\circ} 2161$ is suspected. However, a quantitative analysis is difficult due to significant uncertainty in the continuum definition.
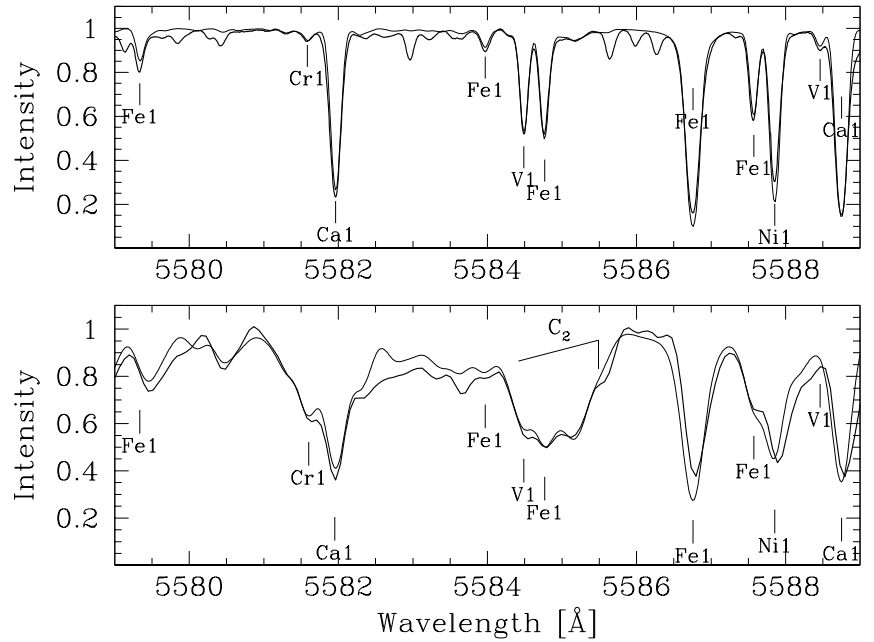

Fig. 4. Same as Fig. 3, but around the $\mathrm{C}_{2}$ Swan system $(1,2)$ bandhead at $5585 \AA$. The thin line represents the synthetic spectrum with carbon abundances of $\log \epsilon(\mathrm{C})=8.70$.

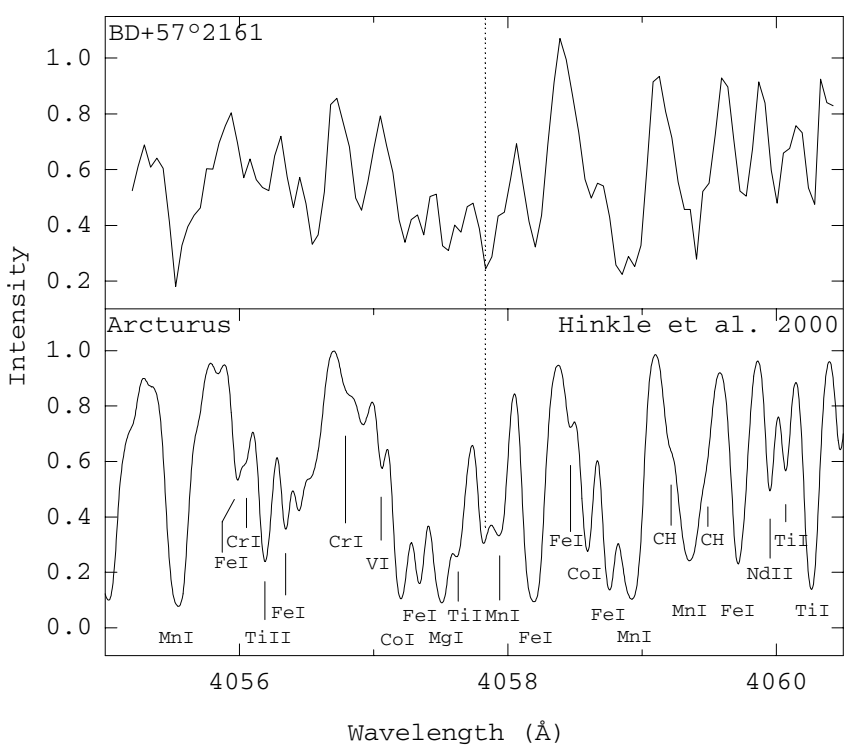

Fig. 5. The observed spectra of $\mathrm{BD}+57^{\circ} 2161$ and the standard star Arcturus around $4057 \AA$ corrected for radial velocities. The dotted line indicates the laboratory wavelength of $\mathrm{Pb}$ I line at $4057.81 \AA$.

\section{Discussion and conclusions}

The atmospheric parameters and metallicity of BD $+57^{\circ} 2161$ are close to those for R-type carbon stars. However, typical Rtype carbon stars are single stars (McClure 1997) and do not show any signatures of s-process enhancement (Dominy 1984). Our radial-velocity measurements suggest the status of a longperiod binary for $\mathrm{BD}+57^{\circ} 2161$. Therefore mass transfer from an AGB star (now WD) to a less evolved companion in the past is a good explanation of the abundance peculiarities detected. However, the [hs/ls] ratio in the atmosphere of the analyzed star is clearly higher than has been found for typical disk binaries. Another difference is the high carbon $(\mathrm{C} / \mathrm{O})$ and nitrogen abundance. Notice that classical barium stars usually do not display significant features of carbon-bearing molecules in their spectra; as a rule $\mathrm{C} / \mathrm{O}<0.6$ (see, for example, Barbuy et al. 1992). 
On the other hand, the classical halo binaries, the $\mathrm{CH}$ stars, are high-velocity objects with large [hs/ls] ratios of $\sim+0.9$ dex on average (Vanture 1992b). Also notice that another star of the group, $\mathrm{BD}+75^{\circ} 348$, has characteristics similar to those of $\mathrm{BD}+57^{\circ} 2161$ (Začs et al. 2000). In addition, the orbital period and mass function of $\mathrm{BD}+75^{\circ} 348$ (see Sect. 3.1.2) are typical of barium-like binaries. Therefore those two carbon-rich stars could be analogues of the classical $\mathrm{CH}$ and barium stars in the old disk population. Medium-high [hs/ls] ratios for such stars are in agreement with theoretical predictions, because at lower metallicities the heavy s-process elements have higher abundances than the light ones (Busso et al. 2000).

Acknowledgements. We thank Roger Griffin for radial velocity monitoring of $\mathrm{BD}+75^{\circ} 348$, calculation of the orbital elements, and valuable comments. The referee, H.Van Winckel, is thanked for his valuable comments on the paper. This research was financed (for L.Z.) in part from the Latvian Council of Science (grant No. 01.0422). The collaborative program between Latvian and Polish Academy of Sciences and the Mutual Fund of scientific collaboration among Taiwan (ROC), Latvia, and Lithuania is thanked for its support. G.A.G. and F.A.M. are grateful to the Russian Foundation for Basic Research (under the grant No. 02-02-17423) and the Federal Program of Astronomy for financial support. G.G. is grateful to the Korean MOST (Ministry of Science and Technology; grant M1-022-00-0005) and KOSFT for providing an opportunity to work at KAO through the Brain Pool program.

\section{References}

Amiot, C. 1983, ApJS, 52, 329

Barbuy, B., Jorissen, A., Rossi, S. C. F., \& Arnould, M. 1992, A\&A, 262,216

Bartkevicius, A., \& Lazauskaite, R. 1996, BaltA, 5, 1

Bauschlicher, Jr. C. W., Langhoff, S. R., \& Taylor, P. R. 1988, ApJ, 332,531

Bergeat, J., Knapik, A., \& Rutily, B. 2001, A\&A, 369, 178

Bergeat, J., Knapik, A., \& Rutily, B. 2002, A\&A, 390, 967

Busso, M., Gallino, R., \& Wasserburg, G. J. 1999, ARA\&A, 37, 239

Cooper, D. M. 1981, Quantum Spec. Rad. Transfer, 26, 113

Dominy, J. F. 1984, ApJS, 55, 27
ESA 1997, The Hipparcos and Tycho Catalogues, ESA SP-1200

Gustafsson, B., \& Bell, R. A. 1979, A\&A, 74, 313

Hinkle, K., Wallace, L., Valenti, J., \& Harmer, D. 2000, Visible and Near Infrared Atlass of the Arcturus Spectrum 3727-9300 A (San Francisco: ASP)

Jørgensen, U. G., \& Larsson, M. 1990, A\&A, 238, 424

Kelleher, D. E, Mohr, P. J., Martin, W. C., et al. 1999, In Proc. SPIE, 3818,170

Knapp, G., Pourbaix, D., \& Jorissen, A. 2001, A\&A, 371, 222

Knowles, P. J., Werner, H. J., Hay, P. J., \& Cartwright, D. C. 1988, J. Chem. Phys., 89, 7334

Kovács, I. 1969, Rotational Structure in the Spectra of Diatomic Molecules (London: Adam Hilger)

Kurucz, R. L. 1992, in The Stellar Populations of Galaxies, ed. B. Barbuy, \& A. Renzini (Dordrecht: Kluwer), IAU Symp., 149, 225

Kurucz, R. L. 1993, CD-ROM 13 \& 18, Smithsonian Astrophys. Obs. Lambert, D. L. 1978, MNRAS, 182, 249

McClure, R. D., \& Woodsworth, A. W. 1990, ApJ, 352, 709

Musaev, F. A., Galazutdinov, G. A., Sergeev, A. V., et al. 1999, Kinematika i Fizika Nebesnyh Tel, 15, 282

Piskunov, N. E., Kupka, F., Ryabchikova, T. A., Weiss, W. W., \& Jeffery, C. S. 1995, A\&AS, 112, 525

Tsuji, T. 1973, A\&A, 23, 411

Ulla, A., Thejll, P., Kipper, T., \& Jørgensen, U. G. 1997, A\&A, 319, 244

Upgren, A., Sperauskas, J., \& Boyle, R. P. 2002, BaltA, 11, 91

Van Eck, S., Goriely, S., Jorissen, A., \& Plez, B. 2001, Nature, 412, 793

Vandervort, G. L. 1958, AJ, 63, 477

Vanture, A. D. 1992a, AJ, 104, 1986

Vanture, A. D. 1992b, AJ, 104, 1997

Vanture, A. D. 1992c, AJ, 103, 2035

Wallerstein, G., \& Knapp, G. R. 1998, ARA\&A, 36, 369

Yamashita, Y. 1972, Ann. Tokyo Astron. Obs., 13, 169

Yamashita, Y. 1975, PASJ, 27, 325

Yamashita, Y., Nishimura, S., Shimizu, M., et al. 1977, PASJ, 29, 731

Začs, L., Musaev, F. A., Bikmaev, I. F., \& Alksnis, O. 1997, A\&AS, 122,31

Začs, L., Nissen, P. E., \& Schuster, W. J. 1998, A\&A, 337, 216

Začs, L., Schmidt, M. R., \& Schuster, W. J. 2000, A\&A, 358, 1022 